\title{
Degradation and Operation-Aware Framework for the Optimal Siting, Sizing and Technology Selection of Battery Storage
}

\author{
Timur Sayfutdinov, Student Member, IEEE, Charalampos Patsios, Petr Vorobev, Member, IEEE, \\ Elena Gryazina, Member, IEEE, David M. Greenwood, Member, IEEE, Janusz W. Bialek, Fellow, \\ IEEE, and Philip C. Taylor, Senior Member, IEEE
}

\begin{abstract}
This paper addresses the problem of optimal siting, sizing, and technology selection of Energy Storage System (ESS) considering degradation arising from state of charge and Depth of Discharge (DoD). The capacity lost irreversibly due to degradation provides the optimizer with a more accurate and realistic view of the capacity available throughout the asset's entire lifetime as it depends on the actual operating profiles and particular degradation mechanisms. When taking into account the ESS's degradation, the optimization problem becomes nonconvex, therefore no standard solver can guarantee the globally optimal solution. To overcome this, the optimization problem has been reformulated to a Mixed Integer Convex Programming (MICP) problem by substituting continuous variables that cause nonconvexity with discrete ones. The resulting MICP problem has been solved using the Branch-and-Bound algorithm along with convex programming, which performs an efficient search and guarantees the globally optimal solution. We found that the optimal battery use does not necesseraly correspond to it reaching its End of Life state at the end of the service lifetime, which is the result of nonlinear degradation mechanicms from both idling and cycling. Finally, the proposed methodology allows formulating computationally tractable stochastic optimization problem to account for future network scenarios.
\end{abstract}

Index Terms-Battery degradation, convex optimization, energy storage.

\section{NOMENCLATURE}

Sets and indices

$S \quad$ set of future network operation scenarios, indexed by $s$

$T$ set of time intervals, indexed by $t$

I set of generation units, indexed by $i$

$\mathrm{Br}$ set of branches, indexed by $\mathrm{km}$, which denotes a branch connecting nodes $k$ and $m$.

$R \quad$ set of Renewable Energy Sources (RES), indexed by $r$

$K \quad$ set of transmission grid nodes, indexed by $k$ set of Energy Storage (ES) technologies, indexed by $j$

The work was supported by The Engineering and Physical Sciences Research Council, grant EP/N032888/1, Multi-scale Analysis for Facilities for Energy Storage (Manifest) and grant EP/K002252/1 Energy Storage for Low Carbon Grids.

T. Sayfutdinov, P. Vorobev, and E. Gryazina are with the Skolkovo Institute of Science and Technology (Skoltech), Moscow 143025, Russia (e-mail: timur.sayfutdinov@skolkovotech.ru).
$N$ set of charge/discharge cycles, indexed by $n$ Given parameters

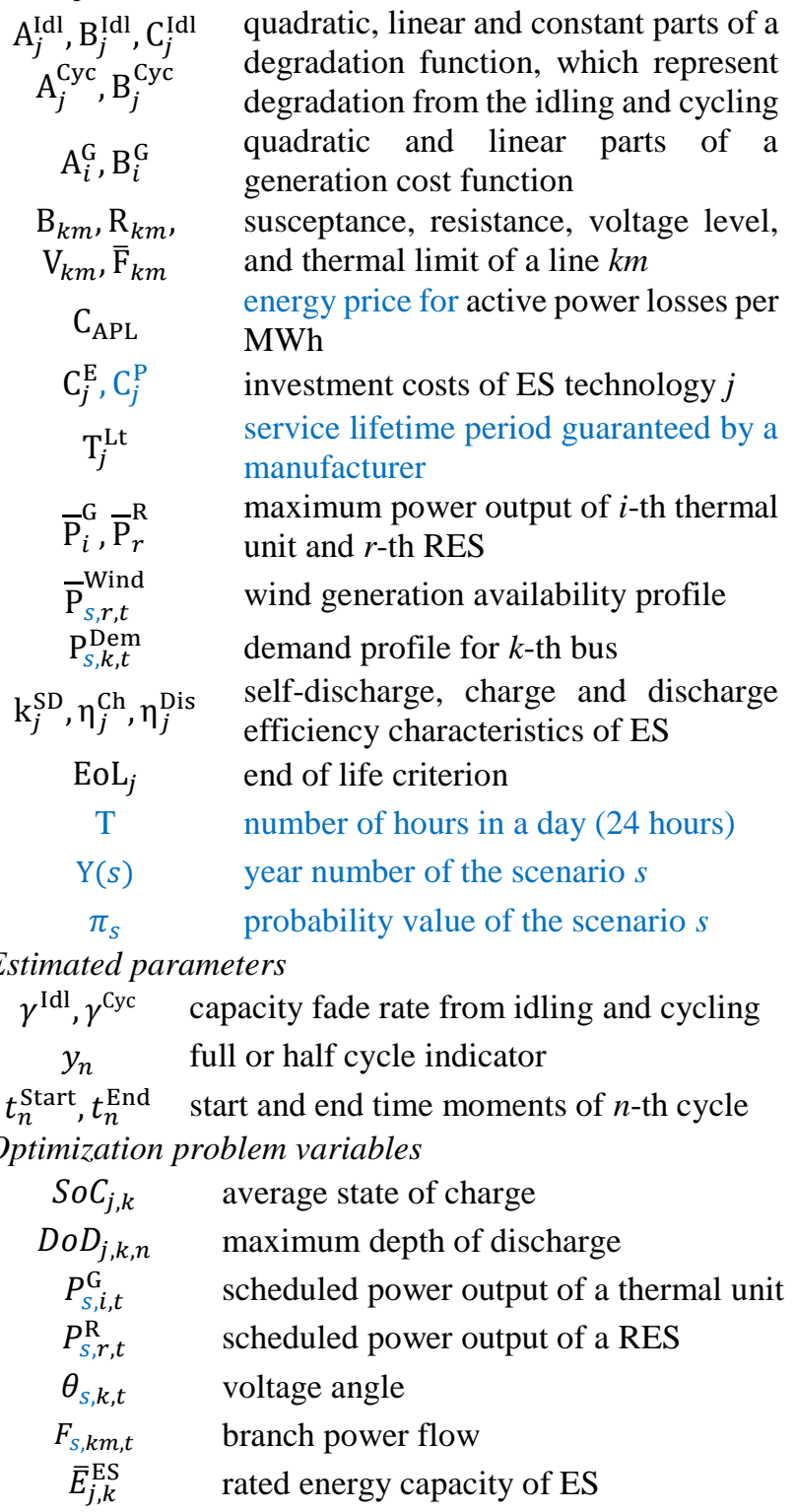

C. Patsios, D. M. Greenwood, and P. C. Taylor are with the School of Engineering, Newcastle University, Newcastle upon Tyne NE1 7RU, U.K. (email: haris.patsios@newcastle.ac.uk).

J. Bialek is with Skolkovo Institute of Science and Technology (Skoltech), Russia (email: j.bialek@skoltech.ru) and with Newcastle University, UK (email: Janusz.bialek@ncl.ac.uk). 


$\begin{array}{cl}\bar{P}_{j, k}^{\mathrm{ES}} & \text { rated power capacity of ES } \\ P_{S, j, k, t}^{\mathrm{ES}+}, P_{S, j, k, t}^{\mathrm{ES}-} & \text { Positive (charge) and negative (discharge) } \\ E_{S, j, k, t}^{\mathrm{ES}} & \text { ES power output } \\ \text { rem }_{j, k} & \text { remarge of ES } \\ & \text { service lifetime }\end{array}$

\section{INTRODUCTION}

$\mathrm{T}^{\mathrm{s}}$ he problem of Siting, Sizing, and Technology selection (SST) for Energy Storage Systems (ESSs) fulfilling power system applications has been considered in many papers. To make the results more accurate, ESS models are applied that account for a variety of ESS characteristics, such as round-trip efficiency, self-discharge, calendar lifetime, operational lifetime, energy to power ratio, degradation, and the End of Life (EoL) criterion. The present study improves solution quality via a degradation aware framework, which incorporates the effects of Depth of Discharge (DoD) and State of Charge (SoC) on battery degradation.

A widely used approach to SST problem consists of formulating it as an optimization problem which has to be efficiently solved even for large systems. Thus, careful selection of mathematical models for representing different battery characteristics is required to keep the optimization problem convex; consequently, it is challenging to incorporate processes like degradation to the overall problem formulation. In [1]-[3] an optimal SST problem is solved considering constant ESS calendar lifetime without accounting for the impact of ESS operation on its lifetime, while [4], [5] consider the total delivered energy by the ESS during the planning horizon while neglecting the inevitable fading of available energy capacity with time. Different approaches to incorporate the capacity fade of the ESS to the optimal SST problem are used in [6]-[12], with an objective of minimizing the operating cost of a network for a finite planning horizon. In [6], [7] a linear energy capacity fade through the calendar lifetime of ESS is considered, while [8] assumes the energy degradation of ESS to be proportional to the total energy throughput of the battery. Number of cycles and SoC dependant degradation is considered in [9] and [10] respectively. In [11] both SoC and power output of ESS are accounted for in degradation process, while [12] considers both idling and cycling degradation mechanisms, where the former is assumed to be constant through time, and the latter is proportional to the energy throughput of the ESS.

Thus, most of the studies on the optimal SST consider the degradation mechanism to be dependent on some single factor, i.e. a linear function of calendar lifetime, energy throughput, number of cycles or SoC. However, the real degradation mechanism of Li-ion batteries is much more complicated - the experimental studies in [13]-[15] identify a number of factors that substantially affect the energy capacity fade: time, cell temperature, the charge/discharge current (C-rate), SoC, and DoD of every cycle. In these papers, the degradation is considered as a cumulative, irreversible process due to both idling and cycling. In [13], [14], [16] sequential algorithms are applied to estimate ESS's degradation for given power output patterns based on measurements of degradation on a set of standard charge/discharge profiles. The degradation from idling is found for an average SoC, and the degradation from cycling is found for each individual cycle and its corresponding DoD which are found by means of the Rainflow Cycle (RFC) counting method [17]. We note, that explicit accounting for operation aware degradation process makes the SST optimization problem neither linear nor convex.

Nonlinearity associated with degradation-aware ESS sizing is dealt with in various ways. In [8], [12] the whole enumeration approach is applied to find the optimal combination of site, size, and technology that gives the least operational cost of a network. In [9] a hybrid heuristic search is applied, where mixed-integer linear programming is used for unit commitment problem, and genetic algorithm is applied for ESS siting and sizing. A stationary degradation map is employed to perform degradation-aware sizing in [11]. In [10] a RFC counting method is applied for ESS sizing and technology selection problem after the optimization process to estimate degradation cost of a candidate solution. The complicated sequential structure of RFC and similar cycle counting methods, which track each cycle and the corresponding DoD value, makes it difficult to apply them within an optimization problem [18]. Standard optimization problem formulations consist of only equalities and inequalities, and sequential algorithms cannot be directly employed. Furthermore, considering the degradation effects from both SoC and DoD results in the nonconvex optimization problem, for which standard solvers cannot guarantee a globally optimal solution.

In the traditional SST problem formulation the battery life is limited by either its service lifetime (usually, around 10-15 years) or the EoL criterion. The latter corresponds to the end of a linear part of Li-ion battery degradation process after which a rapid decrease of available capacity is observed [14] - the value of EoL criterion is technology specific and usually varies around $70 \%-85 \%$ of the initial capacity. In previous papers, where degradation of the battery was considered in the optimization problem formulation, the EoL state was reached exactly at the end of the service lifetime, which is the result of a simplified representation of the degradation process. In the present work we show that the nonlinear degradation dependence on SoC and DoD can result in the final capacity at the end of the service lifetime being higher than the EoL criterion. For example, for one of the case studies (see Appendix A) the highest profitability of the ESS corresponds to the remaining capacity being higher than EoL by $2.4 \%$. This effect is the result of a trade-off between idling and cycling mechanisms of degradation.

In this paper, we propose an optimal SST problem formulation, which extends the state-of-the-art in the area. The optimization problem is designed to find the optimal combination of site, size and technology of an ESS with respect to the optimal power flow, the optimal scheduling of all power generation and consumption units, the optimal battery operation schedule taking into account an accurate degradation model of the Li-ion battery storage as a function of both DoD and SoC. The inherited non-convexity of degradation-aware SST problem has been resolved with Mixed Integer Convex 
Programming (MICP) problem reformulation. Finally, to evaluate the performance of this formulation, the obtained results have been compared to four other approaches, as well as offline performance evaluation.

The original contributions of the research are the following: 1) The optimal siting, sizing, and technology selection problem has been solved accounting for degradation (capacity fade) of the Li-ion battery, considering the effects of both DoD and SoC as part of the optimization process.

2) The optimal solution suggests that the remaining battery capacity at the end of the service lifetime does not necessarily correspond to the EoL criterion, which is the result of the idling and cycling degradation mechanism trade-off.

3) The proposed methodology allows the computationally tractable formulation of a stochastic SST optimization problem to account for future network operation scenarios, i.e. demand, wind, and fuel price scenarios.

\section{MethodolOGY}

\section{A. Degradation Model}

In this paper, we refer to the degradation of an ESS as an integral decrease of energy capacity due to idling and cycling. In [13]-[15] authors identify five stress factors that affect the degradation of Li-ion batteries: time, cell temperature, C-rate, SoC, and DoD of each individual cycle. In the literature, the degradation is divided into two types: idling and cycling. Idling degradation is influenced by the time, the SoC-level during its lifetime and the storage temperature. Cycling degradation is influenced by the number of cycles, the cell temperature, the cycle depth, the average SoC-level during the cycle, and the Crate [13].

Fig. 1 illustrates the capacity fade rate characteristic of Nickel Manganese Cobalt oxide (NMC) Li-ion battery cell during the linear degradation period [15]. Particularly, Fig. 1 (a)

TABLE I

Idling Degradation Data

\begin{tabular}{c|c|c|c|c}
\hline$j$ & Technology & $\mathrm{A}_{j}^{\text {Idl }}$ & $\mathrm{B}_{j}^{\text {Idl }}$ & $\mathrm{C}_{j}^{\text {Idl }}$ \\
\hline 1 & LFP & $6.02 \mathrm{E}-06$ & $1.35 \mathrm{E}-05$ & $1.85 \mathrm{E}-05$ \\
\hline 2 & LMO & $6.81 \mathrm{E}-05$ & $4.02 \mathrm{E}-05$ & $1.63 \mathrm{E}-05$ \\
\hline 3 & NMC & $8.07 \mathrm{E}-06$ & $3.41 \mathrm{E}-06$ & $2.83 \mathrm{E}-05$ \\
\hline 4 & LTO & $3.03 \mathrm{E}-06$ & $2.81 \mathrm{E}-05$ & $5.02 \mathrm{E}-06$ \\
\hline
\end{tabular}

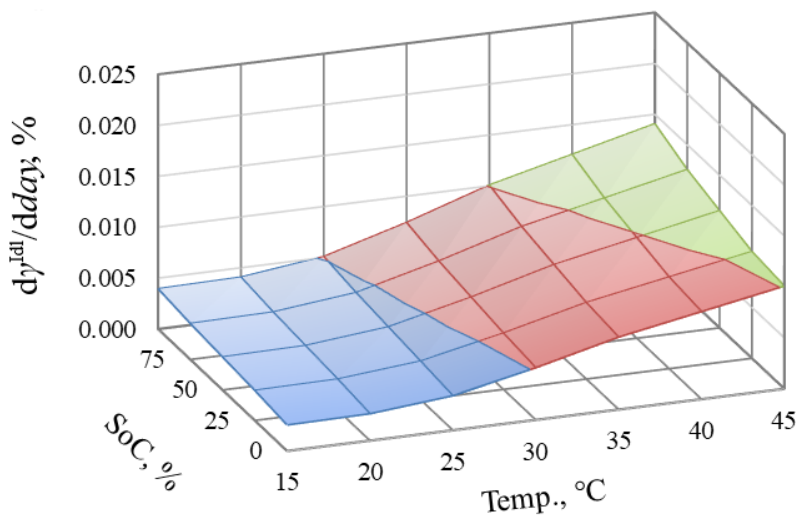

(a) Idling

Fig. 1. Energy capacity fade rate characteristic of Li-ion NMC technology illustrates an amount of capacity lost from idling in one day $\mathrm{d} \gamma^{\text {Idl }} / \mathrm{d}$ day, which is affected by the state of the battery, i.e., SoC and the cell temperature. Fig. 1 (b) illustrates an amount of capacity lost in one cycle at the C-rate less or equal to one $\mathrm{d} \gamma^{\mathrm{Cyc}} / \mathrm{d}$ cycle, which is affected by the DoD of a cycle and the cell temperature. Similar behavior is observed for other types of Li-ion technology. Considering C-rate less or equal to one is reasonable in applications for which the time step unit is greater or equal to one hour, e.g. Energy Time-Shift, as in this paper, meaning that the power output of the ESS cannot exceed one C. Also, considering the C-rate to be less or equal to one allows to consider the cell temperature to be constant because active power losses, which are in a square law with the output current and proportional to the internal cell resistance, are dissipated via a cooling system without significant impact on the cell temperature variation [19]. The latter statement is verified in the results section.

The capacity fade rate characteristics of each type of Li-ion technology, considered within the study, for the C-rate less or equal to one and the cell temperature of $20^{\circ} \mathrm{C}$ are illustrated in Fig. 2. These characteristics have been taken from [13]-[15], [20], and reproduced from the initial nonuniform data by means of quadratic functions as in (1) and (2) using least squares fitting method. The resulting fitting parameters are presented in Table I and II, which are used in the proposed methodology for degradation concerns.

$$
\begin{gathered}
\gamma^{\mathrm{Idl}}\left(\operatorname{SoC}_{j, k}\right)=\mathrm{A}_{j}^{\mathrm{Idl}} S o C_{j, k}{ }^{2}+\mathrm{B}_{j}^{\mathrm{Idl}} S o C_{j, k}+\mathrm{C}_{j}^{\mathrm{Idl}}, \\
\gamma^{\mathrm{Cyc}}\left(D o D_{j, k, n}\right)=\mathrm{A}_{j}^{\mathrm{Cyc}} D o D_{j, k, n}{ }^{2}+\mathrm{B}_{j}^{\mathrm{Cyc}} D o D_{j, k, n},
\end{gathered}
$$

where $S o C_{j, k}$ is an average daily state of charge, $D o D_{j, k, n}$ is a cycle depth of discharge, $\mathrm{A}_{j}^{\mathrm{Idl}}, \mathrm{B}_{j}^{\mathrm{Idl}}, \mathrm{C}_{j}^{\mathrm{Idl}}, \mathrm{A}_{j}^{\mathrm{Cyc}}, \mathrm{B}_{j}^{\mathrm{Cyc}}$ are fitting parameters for the corresponding capacity fade rate characteristics from idling $\gamma^{\text {Idl }}$ and cycling $\gamma^{\text {Cyc }}$.

TABLE II

Cycling Degradation Data

\begin{tabular}{c|c|c|c}
\hline $\mathrm{j}$ & Technology & $\mathrm{A}_{j}^{\text {Cyc }}$ & $\mathrm{B}_{j}^{\text {Cyc }}$ \\
\hline 1 & LFP & $-4.72 \mathrm{E}-05$ & $9.62 \mathrm{E}-05$ \\
\hline 2 & LMO & $-1.21 \mathrm{E}-04$ & $4.01 \mathrm{E}-04$ \\
\hline 3 & NMC & $-4.05 \mathrm{E}-05$ & $1.01 \mathrm{E}-04$ \\
\hline 4 & LTO & $-1.57 \mathrm{E}-05$ & $4.40 \mathrm{E}-05$ \\
\hline
\end{tabular}

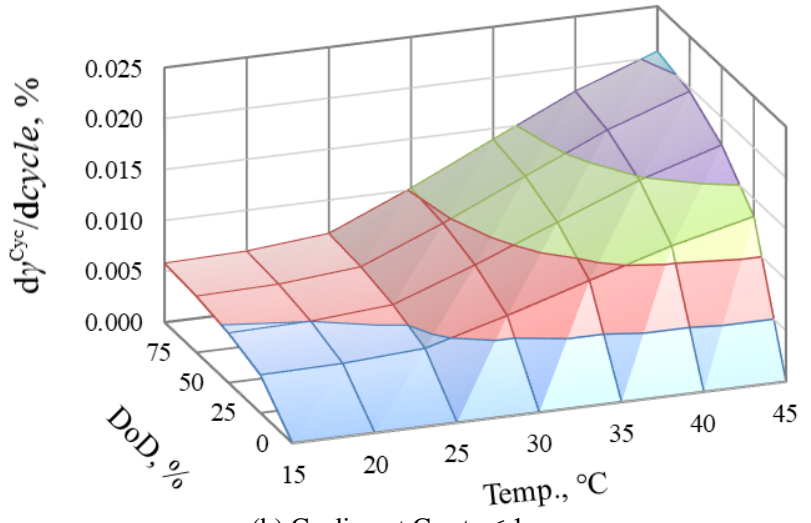

(b) Cycling at C-rate $\leq 1$ 

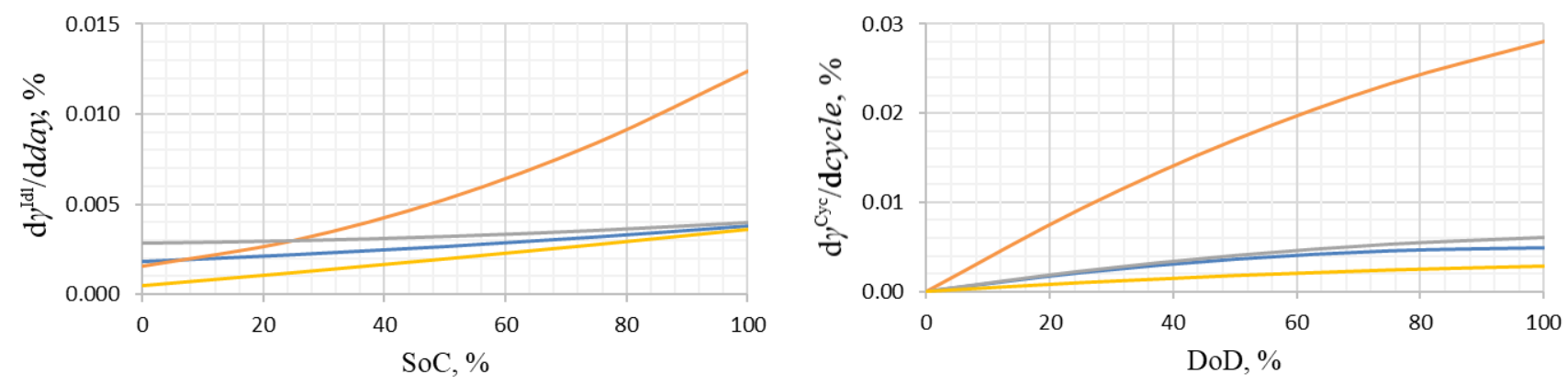

(a) Idling for $20^{\circ} \mathrm{C}$ for a day

$\longrightarrow$ LFP $\longrightarrow$ LMO $\longrightarrow$ NMC

LTO

Fig. 2. Energy capacity fade rate vs. SoC and DoD for fixed cell temperature and C-rate

(b) one cycle for $20^{\circ} \mathrm{C}$ and C-rate $\leq 1$

\section{B. Optimization Problem Formulation: Objective Function}

The stochastic objective function (3) is formulated to find a trade-off between investment cost for ESS and benefits associated with ESS operation. The objective function allows an ESS to perform Energy Time-Shift application, reducing the average daily operational cost of the network over a set of scenarios that represent the whole lifetime horizon of ESS. In (3) the first term yields the total generation costs, the second term represents active power losses within a network, and the last term accounts for the investment cost for the ESS.

$$
\begin{gathered}
\min \sum_{s \in S} \pi_{S} \sum_{t \in T}\left[\sum_{i \in I}\left(\mathrm{~A}_{i}^{\mathrm{G}} P_{S, i, t}^{\mathrm{G}}{ }^{2}-\mathrm{B}_{i}^{\mathrm{G}} P_{S, i, t}^{\mathrm{G}}\right)+\right. \\
\left.+\sum_{k m \in B r}\left(F_{S, k m, t}^{2} \frac{\mathrm{R}_{k m}}{\mathrm{~V}_{k m}^{2}} \mathrm{C}_{\mathrm{APL}}\right)\right] \Delta t+\sum_{k \in K} \sum_{j \in J} \frac{\bar{E}_{j, k}^{\mathrm{ES}} \mathrm{C}_{j}^{\mathrm{E}}+\bar{P}_{j, k}^{\mathrm{ES}} \mathrm{C}_{j}^{\mathrm{P}}}{365 \mathrm{~T}_{j}^{\mathrm{Lt}}},
\end{gathered}
$$

where $S$ is a set of future network operation scenarios indexed by $s, T$ is a set of time intervals indexed by $t$ with a time step $\Delta t$, $I$ is a set of generation units indexed by $i, B r$ is a set of branches indexed by branch connecting pair of nodes $\mathrm{km}, K$ is a set of transmission grid nodes indexed by $k, J$ is a set of energy storage technologies indexed by $j, \pi_{s}$ is a probability value of the scenario $s, P_{s, i, t}^{\mathrm{G}}$ is a scheduled power output of a thermal generation unit, $\mathrm{A}_{i}^{\mathrm{G}}$ and $\mathrm{B}_{i}^{\mathrm{G}}$ are quadratic and linear parts of a generation cost function, $F_{s, k m, t}$ is a branch power flow, $\mathrm{R}_{k m}$ is a branch resistance, $\mathrm{V}_{k m}$ is a branch voltage level, $\mathrm{C}_{\mathrm{APL}}$ is a constant energy price for active power losses per $\mathrm{MWh}, \mathrm{C}_{j}^{\mathrm{E}}$ and $\mathrm{C}_{j}^{\mathrm{P}}$ are investment costs for energy storage per MWh and MW of the installed capacity, $\mathrm{T}_{j}^{\mathrm{Lt}}$ is a constant parameter that defines a service lifetime period guaranteed by a manufacturer, $\bar{E}_{j, k}^{\mathrm{ES}}$ and $\bar{P}_{j, k}^{\text {ES }}$ are rated energy and power capacities of energy storage, i.e. investment decision variables of the optimal SST problem.

The proposed objective function extends the traditional DC Optimal Power Flow (DC OPF) formulation by considering active power losses within the objective function, which are not considered in the power balance constraint as the quadratic dependence of power losses does not meet the affinity requirement for equality constraint in a convex problem formulation. For the same reason, to approximate the value of active power losses while keeping the objective function convex, the energy price for active power losses is considered constant.

\section{Optimization Problem Formulation: Variables}

The optimization problem is solved with respect to the scheduled power output of all thermal units $P_{S, i, t}^{\mathrm{G}}$ and Renewable Energy Sources (RESs) $P_{s, r, t}^{\mathrm{R}}$, the voltage angle at each node $\theta_{S, k, t}$, the branch power flows $F_{S, k m, t}$, the scheduled power output of ESSs - positive (charge) $P_{S, j, k, t}^{\mathrm{ES}+}$ and negative (discharge) $P_{S, j, k, t}^{\mathrm{ES}-}$, the charge of ESSs $E_{S, j, k, t}^{\mathrm{ES}}$, the rated energy and power capacities of each energy storage type at every node $\bar{E}_{j, k}^{\mathrm{ES}}$ and $\bar{P}_{j, k}^{\mathrm{ES}}$, the operational strategy of each ESS $S o C_{j, k}$ and $D o D_{j, k, n}$, and the remaining capacity of ESS at the end of the service lifetime rem $_{j, k}$.

\section{Optimization Problem Formulation: Constraints}

Optimal SST necessitates modeling of a network, generation units, and ESSs; this is done in the constraints of the optimization problem (4)-(21).

Real power production constraints for thermal generation units and RESs are satisfied by inequalities:

$$
\begin{gathered}
-\overline{\mathrm{P}}_{i}^{\mathrm{G}} \leq P_{s, i, t}^{\mathrm{G}} \leq 0 \forall s \in S, i \in I, t \in T, \\
-\overline{\mathrm{P}}_{r}^{\mathrm{R}} \leq P_{s, r, t}^{\mathrm{R}} \leq 0 \forall s \in S, r \in R, t \in T,
\end{gathered}
$$

where $R$ is a set of RESs indexed by $r, \overline{\mathrm{P}}_{i}^{\mathrm{G}}$ and $\overline{\mathrm{P}}_{r}^{\mathrm{R}}$ are the maximum power outputs of a thermal generation unit and a RES correspondingly.

RES power output is also constrained by environmental conditions, i.e. wind availability profile $\overline{\mathrm{P}}_{s, r, t}^{\text {Wind }}$ :

$$
-\overline{\mathrm{P}}_{s, r, t}^{\text {Wind }} \leq P_{s, r, t}^{\mathrm{R}} \leq 0 \forall s \in S, r \in R, t \in T \text {. }
$$

Real power balance at each node is satisfied with equality (7). It has been assumed that energy storage of each type $j$ might be installed at every bus $k$.

$$
\begin{gathered}
\sum_{j \in J}\left(P_{S, j, k, t}^{\mathrm{ES}+}+P_{S, j, k, t}^{\mathrm{ES}-}\right)+P_{S, k, t}^{\mathrm{G}}+P_{S, k, t}^{\mathrm{R}}+\mathrm{P}_{S, k, t}^{\mathrm{Dem}}+ \\
+P_{S, k, t}^{\mathrm{Inj}}=0 \forall s \in S, k \in K, t \in T,
\end{gathered}
$$

where $\mathrm{P}_{S, k, t}^{\mathrm{Dem}}$ is a demand profile, and power generation by a thermal generation unit $P_{s, k, t}^{\mathrm{G}}$ and $\operatorname{RES} P_{s, k, t}^{\mathrm{R}}$ at node $k$ are found as follows:

$$
\begin{gathered}
P_{s, k, t}^{\mathrm{G}}=\left\{\begin{array}{c}
P_{s, i, t,}^{\mathrm{G}}, \text { if } i=k \\
0, \text { if } i \neq k
\end{array}\right. \\
P_{s, k, t}^{\mathrm{R}}=\left\{\begin{array}{c}
P_{s, r, t}^{\mathrm{R}}, \text { if } r=k \\
0, \text { if } r \neq k
\end{array}\right.
\end{gathered}
$$

Total net real power injection at node $k$ is: 


$$
P_{S, k, t}^{\mathrm{Inj}}=\sum_{k m \text { or } m k \in B r} F_{S, k m, t},
$$

where the branch real power flow is found as follows

$$
F_{s, k m, t}=\left(\theta_{s, k, t}-\theta_{s, m, t}\right) \mathrm{B}_{k m},
$$

and $\mathrm{B}_{k m}$ is a branch susceptance.

The real power flow for every branch is limited by its thermal limit $\overline{\mathrm{F}}_{k m}$ as follows:

$$
-\overline{\mathrm{F}}_{k m} \leq F_{s, k m, t} \leq \overline{\mathrm{F}}_{k m} \forall s \in S, k m \in B r, t \in T .
$$

Charge and discharge power outputs are limited by the ESS power rating as follows:

$$
\begin{gathered}
0 \leq P_{S, j, k, t}^{\mathrm{ES}+} \leq \bar{P}_{j, k}^{\mathrm{ES}} \forall s \in S, j \in J, k \in K, t \in T, \\
-\bar{P}_{j, k}^{\mathrm{ES}} \leq P_{s, j, k, t}^{\mathrm{ES}-} \leq 0 \forall s \in S, j \in J, k \in K, t \in T .
\end{gathered}
$$

Energy storage continuity constraint is formulated as follows:

$$
\begin{gathered}
E_{s, j, k, t+1}^{\mathrm{ES}}=\left(1-\mathrm{k}_{j}^{\mathrm{SD}}\right) E_{s, j, k, t}^{\mathrm{ES}}+ \\
+\left(\eta_{j}^{\mathrm{Ch}} P_{S, j, k, t}^{\mathrm{ES}+}+\frac{P_{s, j, k, t}^{\mathrm{ES}-}}{\eta_{j}^{\text {Dis }}}\right) \Delta t \forall s \in S, j \in J, k \in K, t \in T,
\end{gathered}
$$

where self-discharge $k_{j}^{S D}$, charge $\eta_{j}^{\text {Ch }}$ and discharge $\eta_{j}^{\text {Dis }}$ efficiencies are taken into account.

The net daily energy charge is set to zero, which is essential when considering daily scenarios independently:

$$
E_{S, j, k, 1}^{\mathrm{ES}}-E_{S, j, k, T+1}^{\mathrm{ES}}=0 \forall s \in S, j \in J, k \in K .
$$

The charge of ESS is limited by the corresponding energy rating concerning its capacity fade through the lifetime horizon:

$$
0 \leq E_{s, j, k, t}^{\mathrm{ES}} \leq \bar{E}_{j, k}^{\mathrm{ES}}\left[1-\left(\gamma^{\mathrm{Idl}}\left(\operatorname{SoC}_{j, k}\right)+\sum_{n \in N} y_{n} \gamma^{\mathrm{Cyc}}\left(D o D_{j, k, n}\right)\right) \mathrm{Y}(s)\right]
$$

$$
\forall s \in S, j \in J, k \in K, t \in T,
$$

where $N$ is a set of charge/discharge cycles indexed by $n, \gamma^{\text {Idl }}$ and $\gamma^{\mathrm{Cyc}}$ are capacity fade rate characteristics defined in (1) and (2), $y_{n}$ takes either 1.0 or 0.5 , indicating full and half cycles respectively, and $\mathrm{Y}(s)$ is a year number of the scenario $s$.

The remaining capacity of ESS at the end of the service lifetime is found by the degradation caused by idling, cycling, and chosen operation strategy:

$$
\operatorname{rem}_{j, k}=1-\left(\gamma^{\mathrm{Idl}}\left(S o C_{j, k}\right)+\sum_{n \in N} y_{n} \gamma^{\mathrm{Cyc}}\left(D o D_{j, k, n}\right)\right) \mathrm{T}_{j}^{\mathrm{Lt}}
$$

To ensure that the remaining capacity does not get below the EoL threshold, which indicates the end of the linear part of degradation process, the following inequality is applied:

$$
\mathrm{EoL}_{j} \leq \operatorname{rem}_{j, k} \leq 1, \forall j \in J, k \in K,
$$

where $\mathrm{EoL}_{j}$ is a constant parameter that defines the EoL criterion of a particular technology.

In the proposed problem formulation, $S o C_{j, k}$ and $D o D_{j, k, n}$ are considered as targets in the operational strategy, hence, inequalities in (20) and (21) are applied:

$$
\begin{gathered}
\frac{1}{\mathrm{~T}} \bar{E}_{j, k}^{\mathrm{ES}} \sum_{t \in T} E_{S, j, k, t}^{\mathrm{ES}} \Delta t \leq S o C_{j, k}, \forall s \in S, j \in J, k \in K, \\
\frac{1}{2 \bar{E}_{j, k}^{\mathrm{ES}}} \sum_{t=t_{n}^{\mathrm{start}}}^{t_{n}^{\mathrm{End}}}\left(P_{S, j, k, t}^{\mathrm{ES}+}-P_{S, j, k, t}^{\mathrm{ES}-}\right) \Delta t \leq D o D_{j, k, n}, \\
\forall S \in S, n \in N, j \in J, k \in K,
\end{gathered}
$$

where $\mathrm{T}$ is a number of hours in a day, $t_{n}^{\text {Start }}$ and $t_{n}^{\text {End }}$ are the start and the end time moments of a particular cycle.

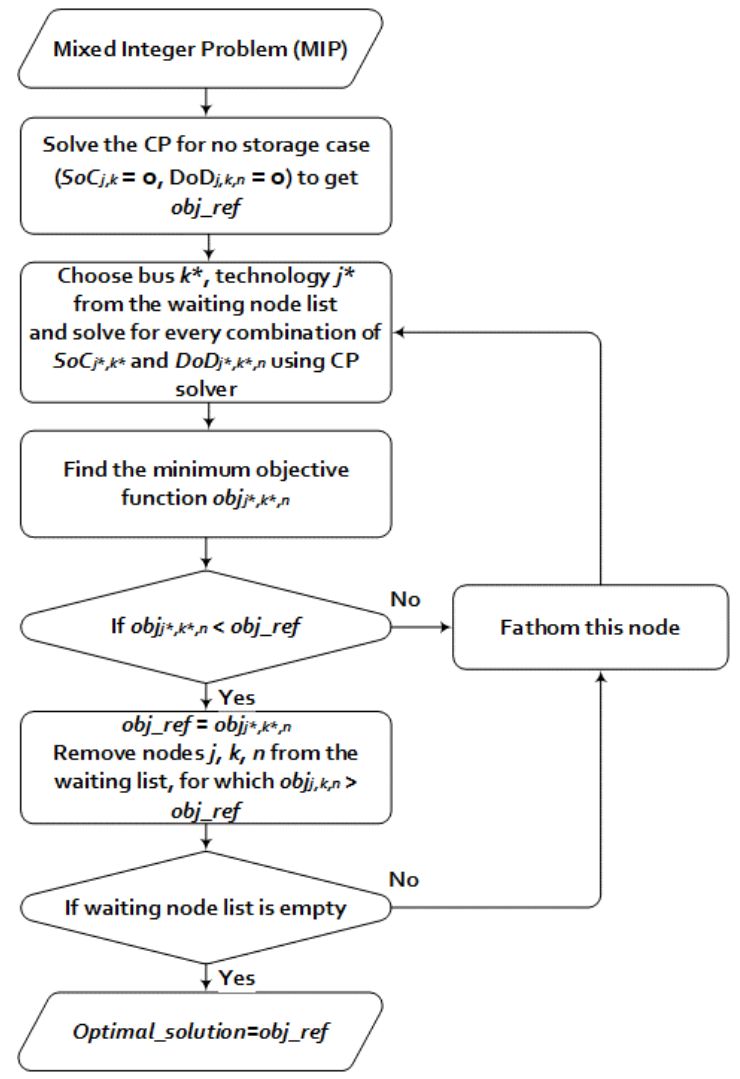

Fig. 3. Flow chart of the Branch-and-Bound algorithm

The average daily SoC in (20) is found as the average daily charge divided by the energy rating, while the cycle DoD value in (21) is found as an energy throughput during a cycle divided by double energy rating because a cycle implies one charge and one discharge. The inequality (21) allows reformulation of sequentially structured cycle counting methods, i.e. RFC, if the start and the end time moments of each cycle are predefined. As it is shown further in sections III and IV, the plausible suggestions for the start and the end time moments for each of the cycles can be made based on the demand profile.

\section{E. MIP Problem Formulation}

When considering both sizing and degradation at the same time, the resulting optimization problem is neither linear nor convex. Specifically, as it can be seen from inequality (17), the rated energy capacity variable $\bar{E}_{j, k}^{\mathrm{ES}}$ is multiplied by the capacity fade rate characteristics $\gamma^{\mathrm{Idl}}$ and $\gamma^{\mathrm{Cyc}}$, which are convex function (1) from $S o C_{j, k}$ and concave function (2) from $D o D_{j, k, n}$ respectively, as depicted in Fig. 2. Also, inequalities (20) and (21) contain a product of variables and fail to meet requirements for convex problem formulation. Standard numerical approaches do not guarantee the global optimum solution for this kind of problem. To overcome this, we propose to substitute continuous variables $S o C_{j, k}$ and $D o D_{j, k, n}$, which are the cause of nonconvexity, with integer variables. Therefore, the nonconvex continuous problem becomes a MICP, where the optimization problem possesses the property of convexity for the fixed $S o C_{j, k}$ and $D o D_{j, k, n}$.

The brute force method to find the optimal solution is to solve the convex optimization problem for every combination of discrete $S o C_{j, k}$ and $D o D_{j, k, n}-\mathrm{a}$ whole enumeration approach. 


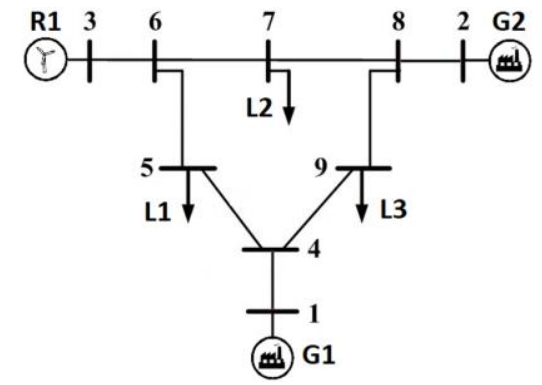

Fig. 4. Case Study Network

However, the whole enumeration approach is very demanding in terms of computational burden. One of the ways to reduce computational burden is to apply the Branch-and-Bound algorithm, which is defined as partial enumeration procedure employing tests of feasibility and comparison to an incumbent solution to fathom candidate problems [21]. A flow chart illustrating the Branch-and-Bound algorithm is shown in Fig. 3.

\section{CASE STUdY}

\section{A. Case Study Network}

The methodology presented in the previous section will now be demonstrated on a slightly modified IEEE nine-bus transmission network, which is depicted in Fig. 4. The generation unit at node three has been replaced with a wind turbine to incorporate renewable generation into the case study.

\section{B. Generation Data}

The case study network contains two conventional thermal generation units and one renewable energy source. Location, maximum power output and cost data for the generation units are presented in Table III. The operation cost of a RES is considered negligible to ensure that it produces the maximum power available.

The generation cost of a thermal generation unit is considered to be a quadratic function:

$$
C_{i, t}^{\mathrm{G}}=\mathrm{A}_{i}^{\mathrm{G}} P_{i, t}^{\mathrm{G}^{2}}+\mathrm{B}_{i}^{\mathrm{G}} P_{i, t}^{\mathrm{G}} .
$$

TABLE III Generators' Data

\begin{tabular}{c|c|c|c|c}
\hline Name & Bus & $\begin{array}{c}\text { Pmax, } \\
\text { MW }\end{array}$ & $\begin{array}{c}\mathrm{A}_{i}^{\mathrm{G}}, \\
\mathrm{f} / \mathrm{MW}^{2}\end{array}$ & $\begin{array}{c}\mathrm{B}_{i}^{\mathrm{G}}, \\
\mathrm{f} / \mathrm{MW}\end{array}$ \\
\hline $\mathrm{G} 1$ & 1 & 250 & 0.6 & 5 \\
\hline $\mathrm{G} 2$ & 2 & 300 & 0.25 & 1.2 \\
\hline $\mathrm{R} 1$ & 3 & 100 & - & - \\
\hline
\end{tabular}

\section{Energy Storage Characteristics}

Four Li-ion based technologies are considered for the selection between or combination of: $\mathrm{LiFePO}_{4}(\mathrm{LFP}), \mathrm{LiMn}_{2} \mathrm{O}_{4}$ (LMO), $\mathrm{LiNiMnCoO}_{2}$ (NMC), $\mathrm{Li}_{4} \mathrm{Ti}_{5} \mathrm{O}_{12}$ (LTO). The proposed methodology accounts for charge/discharge efficiencies, selfdischarge rate, EoL criterion, the investment costs for battery capacity and inverter power rating. Inverter data and battery characteristics have been taken from [22], [23] and are represented in Table IV.

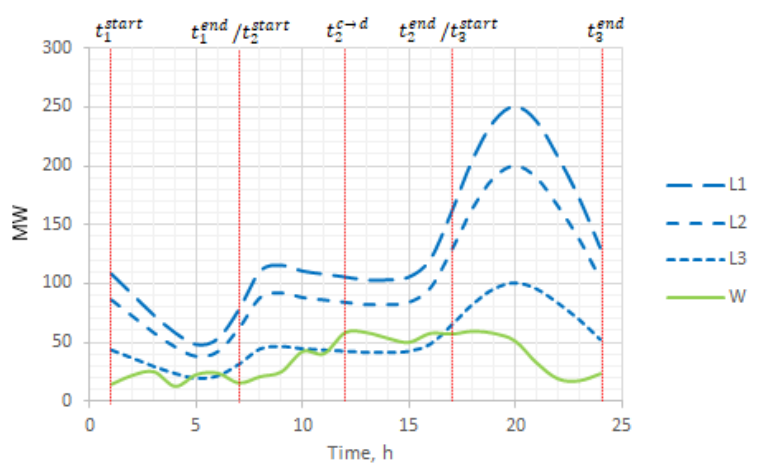

Fig. 5. Wind and demand scenarios.

The degradation characteristics of each type of Li-ion technology are represented in Tables I and II of section II.

TABLE IV

Li-ion Technologies' Characteristics

\begin{tabular}{l|c|c|c|c|c|c}
\hline Tech. & $\begin{array}{c}\text { Disch } \\
\text { eff., } \\
(\%)\end{array}$ & $\begin{array}{c}\text { Ch. } \\
\text { eff., } \\
(\%)\end{array}$ & $\begin{array}{c}\text { Self-dis., } \\
(\% / m o n .)\end{array}$ & $\begin{array}{c}\text { EoL, } \\
\%\end{array}$ & $\begin{array}{c}\text { Battery } \\
\text { Cost, } \\
(£ / k W h)\end{array}$ & $\begin{array}{c}\text { Inverter } \\
\text { Cost, } \\
(£ / k W)\end{array}$ \\
\hline LFP & 97.5 & 97.5 & 4 & 75 & 290 & 90 \\
\hline LMO & 98.5 & 98.5 & 3 & 85 & 250 & 90 \\
\hline NMC & 99 & 99 & 1 & 70 & 270 & 90 \\
\hline LTO & 95 & 95 & 2 & 70 & 770 & 90 \\
\hline
\end{tabular}

\section{Wind and Demand Data}

Wind and demand profiles are considered as input data for the optimization problem. Demand and wind data were taken from the Customer-Led Network Revolution project [24]. Wind and demand profiles are illustrated in Fig. 5. Load profiles L1, L2, L3 represent the demand of the corresponding consumers depicted on Fig. 4, and wind availability profile $\mathrm{W}$ is for renewable generation unit R1.

As it was proposed in section II, demand profiles might be used to formulate the DoD limit constraints (21), avoiding incompatible RFC and similar sequential algorithms. From Fig. 5 , it can be noted that the demand profiles display two peaks and two valleys, which are considered to be the main indicators when the ESS is charging or discharging when performing Energy Time-Shift. The expected state change moments are marked with red dotted lines in Fig. 5. It has been assumed that ESS would perform two half cycles at the beginning [1h; 7h] and at the end [17h;24h] of the day, and one full cycle in the middle of the day [8h; 16h].

\section{RESUlTS AND DISCUSSION}

\section{A. Results}

The optimization problem proposed in section II is formulated for a ten year time horizon, which corresponds to the service lifetime of the storage technologies described in section III. C. Each year is represented by a set of demand and wind scenarios. Particularly, the first year is introduced with a scenario of demand and wind profiles illustrated in Fig. 5. In the following years, to introduce an increasing demand, the original load profiles from Fig. 5 were increased by one percent for each consecutive scenario. To reflect the increasing 


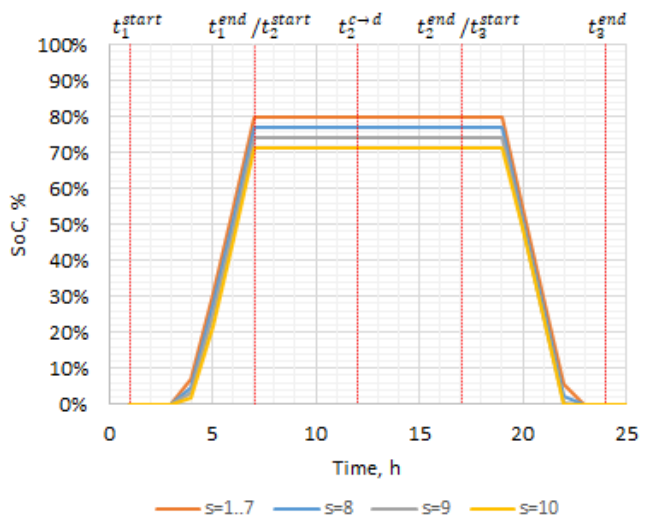

Fig. 6. SoC of NMC ESS at bus 5

renewables penetration, the original wind profile from Fig. 5 was increased by two percent for each consecutive scenario. Integer variables that represent an operational strategy $\operatorname{SoC}_{j, k}$ and $D o D_{j, k, n}$ are defined in the search space $[0.1 ; 1]$ with a discrete step size of 0.1 . The energy price for active power losses is considered to be equal to $50 \mathrm{f} / \mathrm{MWh}$.

The results of the optimization are presented in the last row of Table V. The optimal solution of the SST problem corresponds to $334.33 \mathrm{MWh} / 82.76 \mathrm{MW}$ of $\mathrm{Li}$-ion NMC ESS installed at bus five (co-located with L1). The optimal operating strategy of the installed ESS corresponds to an average SoC equal to $50 \%$ and DoD equal to $80 \%$ for the two considered half cycles $\mathrm{DoD}_{1}$ and $\mathrm{DoD}_{3}$. The $\mathrm{DoD}$ of the full cycle $\mathrm{DoD}_{2}$ is $0 \%$ meaning that no cycle is performed.

To make sure that the time frames proposed for the charge/discharge cycles in section III coincide with the optimal solution, the ESS SoC profile is presented in Fig. 6 for each scenario set $s \in S$, which represent each consecutive year of ESS operation. Similarly to Fig. 5, the same state change instances for the ESS are marked with red dotted lines. As can be seen from Fig. 6, each of the considered cycles is limited within the proposed time frames, meaning that DoD limit constraints (21) are properly formulated. If they were not, the time frames have to be updated according to the results of the optimization problem. Fig. 6 also shows that during the first seven years of operation ( $s=1 . .7)$ ESS operates at the maximum DoD of $80 \%$ that is dictated by the operational strategy. Starting from the eighth year $(s=8, s=9, s=10)$, the DoD is limited with the remaining capacity of ESS, which drops below $80 \%$ of the initial capacity.
The proposed methodology extends the traditional DC OPF formulation by considering active power losses within the objective function, which contribute to the nonuniform locational marginal price distribution within network nodes, hence, affects the optimal siting decision making. In the demonstrated numerical study the energy price for active power losses is considered to be equal to $50 \mathrm{f} / \mathrm{MWh}$, which is around an average price for energy in the case study. The particular value leads to an error of actual power loss estimation equal to $3.1 \%$.

Finally, the proposed problem formulation assumes a unity power factor. To account for a power factor less than one, branch real power flow $F_{s, k m, t}$ used in (3) and (12) has to be replaced with the apparent power flow, which is found as $F_{s, k m, t} / \cos \varphi$, where $\cos \varphi$ is a power factor. This will not change the optimization procedure, but will lead to slightly different numerical results, with the overall effect of making storage use more profitable.

\section{B. Comparative Analysis}

To evaluate the effect from considering degradation as a function of SoC and $\mathrm{DoD}$, as well as variable remaining capacity in the optimal SST problem, the case study described in the section III has been solved for the same input data and the objective function using four other approaches. To account for the error, associated with degradation estimations and power loss approximation, an accurate post-process degradationaware simulation is applied for the obtained solutions as in [13].

The results of all five approaches are presented in Table V. The first approach is used for a reference, and it corresponds to No Storage case. The second approach - No Degradation case - corresponds to the optimal SST problem in which capacity fade is not taken into account [1]. The third corresponds to the optimal SST problem considering degradation as a linear function of energy throughput [8] - Linear Degradation case. In the fourth approach, the degradation effect is considered to be similar to the one proposed in this paper, where degradation is considered as a function of $\mathrm{DoD}$ and $\mathrm{SoC}$, but the remaining capacity at the end of the battery service lifetime is equal to EoL criterion $-\operatorname{Deg}(\mathrm{SoC}, \mathrm{DoD}), \mathrm{rem}=\mathrm{EoL}$ case. The fifth approach corresponds to the one proposed in the methodology, where degradation is considered as a function of $\mathrm{DoD}$ and $\mathrm{SoC}$, and the remaining capacity at the end of the battery service lifetime is considered as a variable of the optimization problem, which may take any value between EoL criterion and 100\% -

TABLE V

Comparative Study

\begin{tabular}{|c|c|c|c|c|c|c|c|c|c|c|c|}
\hline \multirow[b]{2}{*}{$\#$} & \multirow[b]{2}{*}{ Approach } & \multirow{2}{*}{$\begin{array}{c}\text { Objective } \\
\text { Function, } \\
£ / \text { day }\end{array}$} & \multirow[b]{2}{*}{ Bus } & \multirow[b]{2}{*}{ Technology } & \multirow{2}{*}{$\begin{array}{c}\text { Energy } \\
\text { Capacity, } \\
\text { MWh }\end{array}$} & \multirow{2}{*}{$\begin{array}{c}\text { Power } \\
\text { Capacity, } \\
\text { MW }\end{array}$} & \multicolumn{4}{|c|}{ Optimal strategy, \% } & \multirow{2}{*}{$\begin{array}{c}\text { rem } \\
\%\end{array}$} \\
\hline & & & & & & & $\mathrm{SoC}$ & $\mathrm{DoD}_{1}$ & $\mathrm{DoD}_{2}$ & $\mathrm{DoD}_{3}$ & \\
\hline 1 & No Storage & 405,066 & - & - & - & - & - & - & - & - & - \\
\hline 2 & No Degradation [1] & 383,560 & 5 & LMO & 373.61 & 100.7 & - & - & - & - & - \\
\hline 3 & Linear Degradation [8] & 389,933 & 5 & LFP & 315.47 & 78.87 & - & - & - & - & 75 \\
\hline 4 & $\begin{array}{c}\operatorname{Deg}(\mathrm{SoC}, \mathrm{DoD}), \\
\text { rem }=\mathrm{EoL}\end{array}$ & 392,259 & 5 & NMC & 327.58 & 81.09 & 40 & 70 & 10 & 70 & 70 \\
\hline $5^{A}$ & $\begin{array}{c}\operatorname{Deg}(\mathrm{SoC}, \mathrm{DoD}), \\
\mathrm{EoL} \leq \mathrm{rem} \leq 100 \%\end{array}$ & 390,809 & 5 & NMC & 334.33 & 82.76 & 50 & 80 & 0 & 80 & 71.4 \\
\hline
\end{tabular}

A _ Proposed Methodology 


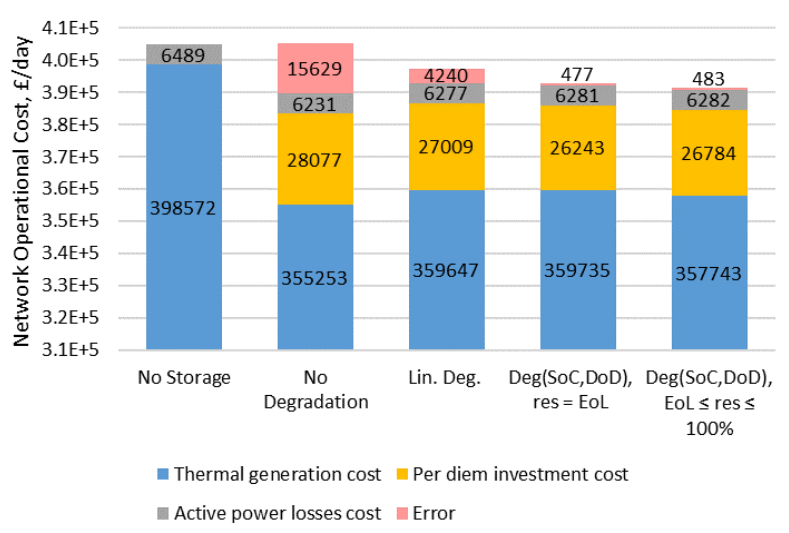

Fig. 7. Objective function stacked chart

$\operatorname{Deg}(\mathrm{SoC}, \mathrm{DoD}), \mathrm{EoL} \leq \mathrm{rem} \leq 100 \%$ case.

A stacked chart of the network operational cost for all cases is illustrated in Fig.7. The maximum investment in ESS occurs in the No Degradation case, where the optimal solution suggests installing $373.61 \mathrm{MWh} / 100.7 \mathrm{MW}$ of LMO technology. This technology is selected because it has the lowest price for capacity. However, when the cost of degradation and the actual power losses are included the estimated daily benefit falls by $£ 15,629$. This shows the importance of taking degradation into account as the optimal investment and operation strategy derived neglecting degradation proves to be inefficient as it completely wipes out benefits from the use of ESS.

The results of the Linear Degradation case suggest installing 315.47MWh/78.87MW of LFP technology. In this case, degradation is proportional to the energy throughput, while the relationships with SoC and DoD are omitted. The degradationaware performance evaluation yields an additional cost of $£ 4,240$, which still makes the use of the ESS beneficial.

The remaining cases, $\operatorname{Deg}(\mathrm{SoC}, \mathrm{DoD})$, rem $=\mathrm{EoL}$, and $\operatorname{Deg}(\mathrm{SoC}, \mathrm{DoD}), \mathrm{EoL} \leq \mathrm{rem} \leq 100 \%$, represent the proposed degradation-aware sizing. The difference between them resides in the fixed and variable remaining capacity at the end of the battery service lifetime. In the first case, where the remaining capacity is tied to EoL, the optimal solution suggests installing 327.58MWh/81.09MW of NMC technology and the error associated with degradation estimation and power loss approximation adds up $£ 477$ to the objective function. When the remaining capacity requirement is relaxed, the optimal solution is to install $334.33 \mathrm{MWh} / 82.76 \mathrm{MW}$ of NMC with the corresponding error of $£ 483$. In both cases, the ESS installation is economically viable.

The difference between the Network Operational Cost of a particular case and the Network Operational Cost of No Storage case from Fig. 7 gives a daily benefit of energy storage use, which is projected to the service lifetime $\mathrm{T}_{j}^{\mathrm{Lt}}$ to get a lifetime benefit. Such that, for the the Linear Degradation case, the lifetime benefit of ESS equals $£ 28.81 \mathrm{M}$. For the fixed remaining capacity case $(\operatorname{Deg}(\mathrm{SoC}, \mathrm{DoD})$, rem $=\mathrm{EoL})$, the lifetime benefit from ESS integration reaches $£ 45 \mathrm{M}$. Variable remaining capacity case delivers a lifetime benefit of $£ 50.27 \mathrm{M}$. Thus, considering a variable remaining capacity at the end of the battery service lifetime rather than setting it equal to the EoL

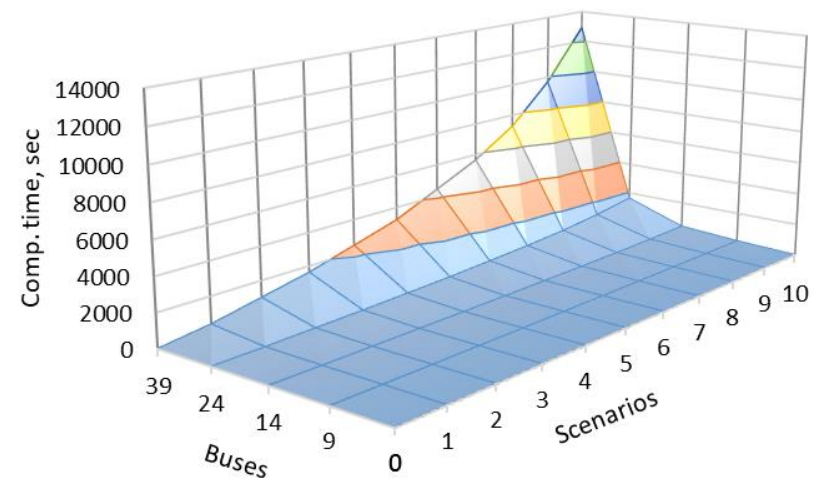

Fig. 8. Computational time vs. number of buses and number of scenarios

criterion increases the profitability of the investment in ESS by $11.7 \%$. According to the results, the optimal remaining capacity at the end of the battery service lifetime is $71.4 \%$, while the EoL criterion for NMC technology is $70 \%$.

\section{Cell Temperature and $C$-rate}

To justify the assumption that cell temperature has a minor effect on the degradation of the ESS for applications where the C-rate is less or equal to one, the scheduled power output, obtained from the optimization problem, has been applied to the thermal model of the Li-ion ESS [15]. The resulting cell temperature varies above $20^{\circ} \mathrm{C}$ by $4.2^{\circ} \mathrm{C}$. According to Fig. 1, the capacity fade rate around $20^{\circ} \mathrm{C}$ is flat and does not change much until $25^{\circ} \mathrm{C}$ and the actual difference in capacity fade is within $1.1 \%$, which makes it possible to conclude that considering limited $\mathrm{C}$-rate and constant cell temperature for the proposed application is a valid simplification.

\section{Scalability}

The proposed Branch-and-Bound algorithm, as well as the optimization problem, have been formulated in JuMP (Julia for Mathematical Optimization). The Ipopt solver has been used to solve the convex optimization problems. The optimization problems have been solved on Intel ${ }^{\circledR}$ Core ${ }^{\mathrm{TM}}$ i5-2410M CPU @ 2.3GHz 4GB RAM laptop computer.

To test the scalability of the proposed problem formulation, it has been solved for various case-study networks and a number of wind and demand scenarios. Particularly, the optimization problem has been solved for the IEEE benchmark systems, i.e., 9-bus, 14-bus, 24-bus, and 39-bus. The network data of which have been taken from the MATPOWER data files [25]. The number of considered wind and demand scenarios varied from one to ten. The results of the ten scenarios cases are provided in the Appendix A.

Fig. 8 illustrates how computational time is affected by the number of scenarios considered, as well as the number of candidate nodes within a network. The increased number of scenarios would only affect the convex part of the optimization problem, the complexity of which is polynomial-time dependent on the number of variables, hence, moderate growth along the number of scenarios axis. However, the number of considered buses affects the combinatorial part of the problem, which has a substantial effect on computational time, hence, results in a rapid increase along the number of buses axis. 


\section{CONCLUSION}

This paper has presented a new battery degradation formulation for use in the optimal siting, sizing, and technology selection of Li-ion battery storage. The considered degradation model - which is dependent on SoC and DoD - has never been used in the literature within an optimization problem due to its sequential structure. In this paper, the degradation model has been reformulated using mathematical expressions to embed it within the optimization problem. The resulting optimization problem became nonconvex, meaning that no standard solver could guarantee the globally optimal solution. To resolve this, the optimization problem has been reformulated to MICP problem by substituting continuous variables that cause nonconvexity with discrete ones. The resulting MICP problem has been solved using the Branch-and-Bound algorithm along with convex programming, which perform an efficient search and guarantee the globally optimal solution. It has been shown that the proposed methodology allows a computationally tractable formulation of a stochastic SST optimization problem to account for various future network operation scenarios.

Considering power losses within the objective function and not in the power balance constraint allows approximating active power losses within the DC OPF framework. However, it is important to note that the approach leads to an underestimation of power generation. The reason for this is that the actual power losses are not modelled within the constraints of the optimization problem, hence, not covered by the generation units. Depending on the value of the energy price for active power losses, which has to be kept constant, the generation costs associated with the power losses may be under- or overestimated. Considering the energy price for active power losses equal to the average price for energy may be a valid simplification if the associated error is acceptable.
The developed methodology has been compared to four other approaches to evaluate the effect of the proposed degradation model, particularly considering the degradation as a function of SoC and DoD. The comparative analysis shows that the proposed methodology performs more rigorous technoeconomic assessment by taking into account degradation from both cycling and idling. The optimal solution does not necessarily correspond to battery reaching its EoL state at the end of the service lifetime. This is the result of a trade-off between idling and cycling degradation mechanicms, when the more profitable solution corresponds to battery operation ensuring slower degradation. This implies that the prospective ESS owner does not have to use the EoL threshold as a reference for determination of optimal battery usage strategy. In the case study considered in this paper, the final capacity of the battery at the end of its service lifetime was $1.4 \%$ higher than the EoL threshold and the profitability of the ESS throughout its lifetime is $11.7 \%$ higher then in the case when EoL criterion is imposed at the end of the service lifetime.

\section{APPENDIX A SST RESULTS}

The SST results for larger networks are provided in the Table A. Depending on the operational strategy and scheduling of ESSs, the optimal remaining capacity value varies from $70.2 \%$ to $72.4 \%$.

\section{REFERENCES}

[1] S. Wogrin and D. F. Gayme, "Optimizing Storage Siting, Sizing, and Technology Portfolios in Transmission-Constrained Networks," IEEE Trans. Power Syst., vol. 30, no. 6, pp. 3304-3313, 2015.

Y. Dvorkin, R. Fernandez-Blanco, D. S. Kirschen, H. Pandzic, J. P. Watson, and C. A. Silva-Monroy, "Ensuring Profitability of Energy Storage," IEEE Trans. Power Syst., vol. 32, no. 1, pp. 611-623, 2017. K. Baker, G. Hug, and X. Li, "Energy Storage Sizing Taking into

TABLE A

SST Results for Various Networks

\begin{tabular}{|c|c|c|c|c|c|c|c|c|c|c|c|c|c|}
\hline \multirow[b]{2}{*}{$\#$} & \multirow[b]{2}{*}{ Network } & \multirow{2}{*}{$\begin{array}{l}\text { Number of } \\
\text { Scenarios }\end{array}$} & \multirow{2}{*}{$\begin{array}{l}\text { Comp. } \\
\text { Time, } \\
\text { seconds }\end{array}$} & \multirow{2}{*}{$\begin{array}{c}\text { Objective } \\
\text { Function, } \\
\text { \&/day }\end{array}$} & \multirow[b]{2}{*}{ Bus } & \multirow[b]{2}{*}{ Techn. } & \multirow{2}{*}{$\begin{array}{c}\text { Energy } \\
\text { Capacity, } \\
\text { MWh }\end{array}$} & \multirow{2}{*}{$\begin{array}{c}\text { Power } \\
\text { Capacity, } \\
\text { MW }\end{array}$} & \multicolumn{4}{|c|}{ Optimal strategy, \% } & \multirow{2}{*}{$\begin{array}{c}\mathrm{rem}, \\
\%\end{array}$} \\
\hline & & & & & & & & & SoC & $\mathrm{DoD}_{1}$ & $\mathrm{DoD}_{2}$ & $\mathrm{DoD}_{3}$ & \\
\hline 1 & $\begin{array}{c}\text { IEEE } \\
\text { 9-bus } \\
\text { System }\end{array}$ & \multirow{21}{*}{10} & 149 & 390,809 & 5 & NMC & 334.33 & 82.76 & 50 & 80 & 0 & 80 & 71.4 \\
\hline \multirow{7}{*}{2} & \multirow{7}{*}{$\begin{array}{c}\text { IEEE } \\
\text { 14-bus } \\
\text { System }\end{array}$} & & \multirow{7}{*}{386} & \multirow{7}{*}{572,308} & 3 & \multirow{7}{*}{ NMC } & 171.9 & 36.4 & 60 & 70 & 0 & 70 & 72.4 \\
\hline & & & & & 7 & & 70.28 & 18.08 & 50 & 90 & 0 & 90 & 70.2 \\
\hline & & & & & 10 & & 27.35 & 5.48 & 60 & 70 & 0 & 70 & 72.4 \\
\hline & & & & & 11 & & 3.8 & 0.99 & 50 & 90 & 0 & 90 & 70.2 \\
\hline & & & & & 12 & & 13.34 & 2.83 & 60 & 70 & 0 & 70 & 72.4 \\
\hline & & & & & 13 & & 31.81 & 6.62 & 60 & 70 & 0 & 70 & 72.4 \\
\hline & & & & & 14 & & 48.55 & 9.45 & 60 & 70 & 0 & 70 & 72.4 \\
\hline \multirow{5}{*}{3} & \multirow{5}{*}{$\begin{array}{c}\text { IEEE } \\
\text { 24-bus } \\
\text { System }\end{array}$} & & \multirow{5}{*}{1,628} & \multirow{5}{*}{$1,738,907$} & 3 & \multirow{5}{*}{ NMC } & 160.17 & 36.41 & 50 & 80 & 0 & 80 & 71.4 \\
\hline & & & & & 6 & & 143.63 & 28.22 & 60 & 70 & 0 & 70 & 72.4 \\
\hline & & & & & 9 & & 150.09 & 32.89 & 50 & 80 & 0 & 80 & 71.4 \\
\hline & & & & & 10 & & 170.81 & 36.88 & 50 & 80 & 0 & 80 & 71.4 \\
\hline & & & & & 14 & & 147.16 & 36.26 & 50 & 80 & 0 & 80 & 71.4 \\
\hline \multirow{8}{*}{4} & \multirow{8}{*}{$\begin{array}{l}\text { IEEE } \\
\text { 39-bus } \\
\text { System }\end{array}$} & & \multirow{8}{*}{12,900} & \multirow{8}{*}{$3,332,499$} & 4 & \multirow{8}{*}{ NMC } & 219.84 & 57.31 & 50 & 90 & 0 & 90 & 70.2 \\
\hline & & & & & 8 & & 389.46 & 98.36 & 50 & 90 & 0 & 90 & 70.2 \\
\hline & & & & & 15 & & 305.5 & 79.64 & 50 & 90 & 0 & 90 & 70.2 \\
\hline & & & & & 18 & & 179.59 & 46.82 & 50 & 90 & 0 & 90 & 70.2 \\
\hline & & & & & 24 & & 137.3 & 35.79 & 50 & 90 & 0 & 90 & 70.2 \\
\hline & & & & & 27 & & 291.43 & 75.97 & 50 & 90 & 0 & 90 & 70.2 \\
\hline & & & & & 28 & & 86.83 & 22.63 & 50 & 90 & 0 & 90 & 70.2 \\
\hline & & & & & 39 & & 363.47 & 91.33 & 50 & 90 & 0 & 90 & 70.2 \\
\hline
\end{tabular}


Account Forecast Uncertainties and Receding Horizon Operation," IEEE Trans. Sustain. Energy, vol. 8, no. 1, pp. 331-340, 2017.

Alsaidan, A. Khodaei, and W. Gao, "A Comprehensive Battery Energy Storage Optimal Sizing Model for Microgrid Applications," IEEE Trans. Power Syst., vol. 33, no. 4, pp. 3968-3980, 2018.

T. Sayfutdinov, C. Patsios, J. W. Bialek, D. M. Greenwood, and P. C. Taylor, "Incorporating variable lifetime and self-discharge into optimal sizing and technology selection of energy storage systems," IET Smart Grid, vol. 1, no. 1, pp. 11-18, 2018.

[6] T. Qiu, B. Xu, Y. Wang, Y. Dvorkin, and D. S. Kirschen, "Stochastic Multistage Coplanning of Transmission Expansion and Energy Storage," IEEE Trans. Power Syst., vol. 32, no. 1, pp. 643-651, 2017.

[7] H. Alharbi and K. Bhattacharya, "Stochastic Optimal Planning of Battery Energy Storage Systems for Isolated Microgrids," IEEE Trans. Sustain. Energy, vol. 9, no. 1, pp. 211-227, 2018.

[8] I. Miranda, N. Silva, and H. Leite, "A Holistic Approach to the Integration of Battery Energy Storage Systems in Island Electric Grids with High Wind Penetration," IEEE Trans. Sustain. Energy, vol. 7, no. 2, pp. 775-785, 2016.

[9] B. Li, R. Roche, D. Paire, and A. Miraoui, "Sizing of a stand-alone microgrid considering electric power, cooling / heating, hydrogen loads and hydrogen storage degradation," Appl. Energy, vol. 205, pp. 1244-1259, 2017.

[10] T. Dragičević, H. Pandžić, D. Škrlec, I. Kuzle, J. M. Guerrero, and D. S. Kirschen, "Capacity Optimization of Renewable Energy Sources and Battery Storage in an Autonomous Telecommunication Facility," IEEE Trans. Sustain. Energy, vol. 5, no. 4, pp. 1367-1378, 2014.

[11] P. Fortenbacher, A. Ulbig, and G. Andersson, "Optimal Placement and Sizing of Distributed Battery Storage in Low Voltage Grids Using Receding Horizon Control Strategies," IEEE Trans. Power Syst., vol. 33, no. 3, pp. 2383-2394, 2018.

[12] H. C. Hesse, R. Martins, P. Musilek, M. Naumann, C. N. Truong, and A. Jossen, "Economic Optimization of Component Sizing for Residential Battery Storage Systems," Energies, vol. 10, no. 7, p. 835, 2017.

[13] D. I. Stroe, M. Swierczynski, A. I. Stroe, R. Laerke, P. C. Kjaer, and R. Teodorescu, "Degradation Behavior of Lithium-Ion Batteries Based on Lifetime Models and Field Measured Frequency Regulation Mission Profile," IEEE Trans. Ind. Appl., vol. 52, no. 6, pp. 50095018, 2016

[14] B. Xu, A. Oudalov, A. Ulbig, G. Andersson, and D. S. Kirschen, "Modeling of Lithium-Ion Battery Degradation for Cell Life Assessment," IEEE Trans. Smart Grid, vol. 9, no. 2, pp. 1131-1140, 2018.

[15] S. F. Schuster et al., "Nonlinear aging characteristics of lithium-ion cells under different operational conditions," J. Energy Storage, vol. 1, pp. 44-53, 2015.

[16] Y. Du, R. Jain, and S. M. Lukic, "A novel approach towards energy storage system sizing considering battery degradation," in IEEE Energy Conversion Congress and Exposition, Proceedings, 2016, pp. $1-8$.

[17] M. Matsuishi and T. Endo, "Fatigue of metals subjected to varying stress," Japan Soc. Mech. Eng., vol. 68, no. 2, pp. 37-40, 1968.

[18] I. Rychlik, "A new definition of the rainflow cycle counting method," Int. J. Fatigue, vol. 9, no. 2, pp. 119-121, 1987.

[19] F. He, A. A. Ams, Y. Roosien, W. Tao, B. Geist, and K. Singh, "Reduced-order Thermal Modeling of Liquid-cooled Lithium-ion Battery Pack for EVs and HEVs," in 2017 IEEE Transportation Electrification Conference and Expo (ITEC), 2017, pp. 507-511.

[20] E. Namor, D. Torregrossa, F. Sossan, R. Cherkaoui, and M. Paolone, "Assessment of Battery Ageing and Implementation of an Ageing Aware Control Strategy for a Load Leveling Application of a Lithium Titanate Battery Energy Storage System," in 2016 IEEE 17th Workshop on Control and Modeling for Power Electronics (COMPEL), 2016, pp. 1-6.

[21] G. R. Parker and R. L. Rardin, Discrete Optimization. Elsevier, 2014.

[22] X. Hu, C. Zou, C. Zhang, and Y. Li, "Technological Developments in Batteries: A Survey of Principal Roles, Types, and Management Needs," IEEE Power Energy Mag., vol. 15, no. 5, pp. 20-31, 2017.

[23] R. Fu, T. Remo, R. Margolis, R. Fu, T. Remo, and R. Margolis, "2018 U . S . Utility-Scale Photovoltaics- Plus-Energy Storage System Costs Benchmark," 2018.

[24] "Customer-Led Network Revolution," 2016. [Online]. Available: http://www.networkrevolution.co.uk/resources/project-data/.
[Accessed: 20-Dec-2018].

[25] R. D. Zimmerman, C. E. Murillo-sánchez, R. J. Thomas, L. Fellow, and A. M. Atpower, "MATPOWER: Steady-State Operations , Systems Research and Education," IEEE Trans. Power Syst., vol. 26, no. 1, pp. 12-19, 2011.

\section{BIOGRAPHIES}

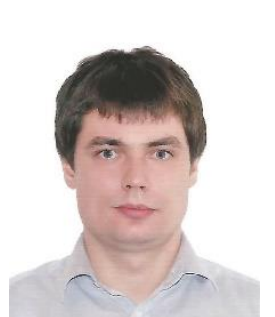

Timur Sayfutdinov (S'19) received the M.Eng. in control in technical systems from Saint-Petersbourg State Polytechnical University, Russia, in 2013. Currently, he is a $\mathrm{PhD}$ student at Skolkovo Institute of Science and Technology, Russia. His research interests include energy storage applications in power system and advanced control strategies in smart grids.

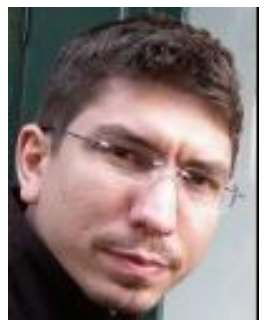

Dr Charalampos (Haris) Patsios is a Senior Lecturer in Power Systems in the School of Engineering at Newcastle University. He obtained his electrical engineering degree in 2005 from the University of Patras and his PhD degree in 2011 from the National Technical University of Athens. He has significant experience in the design, modelling and control of electrical power systems including Energy Storage, Renewables and Power Electronics. His research involves the development of models, grid interfaces and control techniques for energy storage systems as well as decentralized control in future power networks, working closely with UK industry and academia.

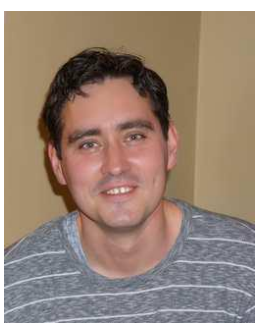

Petr Vorobev (M‘15) received his Ph.D. degree in theoretical physics from Landau Institute for Theoretical Physics, Moscow, in 2010. Currently, he is an Assistant Professor at Skolkovo Institute of Science and Technology (Skoltech), Moscow, Russia. Before joining Skoltech, he was a Postdoctoral Associate at the Mechanical Engineering Department of Massachusetts Institute of Technology (MIT), Cambridge. His research interests include a broad range of topics related to power system dynamics and control. This covers low frequency oscillations in power systems, dynamics of power system components, multitimescale approaches to power system modelling, development of plug-and-play control architectures for microgrids.

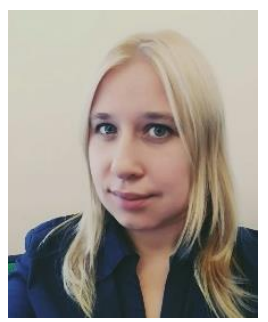

Elena Gryazina received the Ph.D degree from Institute for Control Sciences Russian Academy of Sciences. She is currently an Assistant Professor with Skolkovo Institute of Science and Technology, Moscow, Russia. Her research interests include convex and nonconvex optimization with applications to energy systems engineering problems such as optimal power flow and energy efficient microclimate control in buildings. 


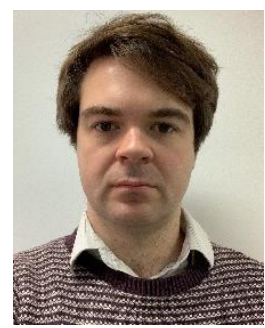

David M. Greenwood (M'14) received the M.Eng. degree in new and renewable energy from Durham University, Durham, U.K., in 2010 and the Ph.D. degree, for work on real-rime thermal ratings, from Newcastle University, Newcastle upon Tyne, U.K., in 2014. He is currently an Academic Track Fellow at Newcastle University. His research focusses on whole systems approaches to energy.

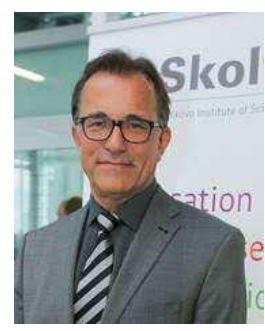

Janusz W. Bialek (F'11) received M.Eng. and Ph.D. degrees from Warsaw University of Technology, Poland. He currently holds joint appointments as Full Professor at Skoltech (Russia) and Professor of Power and Energy Systems at Newcastle University (UK). Previously he has held Chair Professor positions at the University of Edinburgh (2003-2009) and Durham University (2009-2014). His interests are in smart grids, energy system integration, power system dynamics, power system control, power system economics, and preventing electricity blackouts.

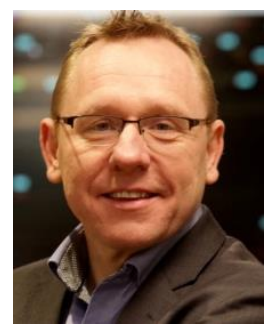

Philip C. Taylor (SM'12) received the Engineering Doctorate in the field of intelligent demand side management techniques from the University of Manchester Institute of Science and Technology (UMIST), Manchester, U.K., in 2001. He joined Newcastle University, Newcastle upon-Tyne, U.K., in April 2013 where he is the Head of the School of Engineering and holds the Siemens Chair of Energy Systems. He is a visiting Professor at Nanyang Technological University in Singapore and he previously held the DONG Energy Chair in Renewable Energy and was a Director of the Durham Energy Institute. 